\title{
Decreasing glioma recurrence through adjuvant cancer stem cell inhibition
}

\author{
This article was published in the following Dove Press journal: \\ Biologics:Targets and Therapy \\ 19 June 2010 \\ Number of times this article has been viewed
}

\author{
Josh Neman \\ Rahul Jandial \\ Division of Neurosurgery, City \\ of Hope National Cancer Center, \\ Duarte, CA, USA
}

Correspondence: Josh Neman Division of Neurosurgery, I 500 East Duarte Road, MOB 200IA, Duarte, CA $91010-3000$, USA

Tel + I 6264717100

Fax + I 626 47I 7344

Email jneman@coh.org

\begin{abstract}
Gliomas remain one of the most challenging solid organ tumors to treat and are marked clinically by invariable recurrence despite multimodal intervention (surgery, chemotherapy, radiation). This recurrence perhaps, is as a consequence of the failure to eradicate a tumor cell subpopulation, termed cancer stem cells. Isolating, characterizing, and understanding these tumor-initiating cells through cellular and molecular markers, along with genetic and epigenetic understanding will allow for selective targeting through therapeutic agents and holds promise for decreasing glioma recurrence.
\end{abstract}

Keywords: glioma, cancer stem cells, epigenetic

\section{Introduction}

Malignant gliomas are the most frequent cancers of the central nervous system and remain the most clinically challenging solid organ tumor. ${ }^{1}$ The median survival for glioblastoma multiform patients has been a dismal 12 months, even with surgery, radiation, and chemotherapy. ${ }^{2}$ With the recent use of the DNA alkylating agent temozolomide, the median survival has increased by two months to approximately 14 months. ${ }^{3}$ This small yet significant increase in survival represents the greatest advancement in malignant glioma treatment over the last four decades. Recurrences occur almost exclusively in the brain, both at the site of initial tumor presentation and at distance, for example, the opposite hemisphere, illustrating the tumor's infiltrative nature.

The cancer stem cell (CSC) hypothesis proposes that tumors contain a small subset of cells with stem-like properties, which retain the exclusive ability to self-renew and sustain the growth of the tumor; essentially malignant cells that have stem cell like characteristics. Tumor stem cells were first identified in leukemia, ${ }^{4}$ and more recently also discovered in various solid tumors, including cancers of the brain such as glioblastomas, medulloblastomas and ependymomas. ${ }^{1}$ Brain CSCs, also known as brain tumor stem cells (BTSCs), are defined by (i) the capacity to self-renew, (ii) the ability to initiate brain tumors upon orthotopic implantation in xenografts and (iii) multipotency, that is, the capacity to differentiate into cells with a neuronal, astrocytic or oligodendroglial phenotype. ${ }^{5}$ In addition, BTSCs are characterized by the expression of neural stem cell antigens and the ability to grow as nonadherent three dimensional aggregates termed neurospheres when cultured in the presence of epidermal growth factor (EGF) and fibroblast growth factor (FGF) under serum-free conditions. BTSCs possess many molecular and functional similarities with normal neural stem cells (NSCs), supporting the hypothesis that brain tumors could arise 
from neural stem or progenitor cells. ${ }^{6,7}$ Regardless of whether BTSCs arise from normal NSCs, it is known that once brain tumors develop these BTSCs evince properties fundamentally unique from the majority of malignant cells that comprise the bulk of the tumor. BTSCs are resistant to current radiotherapy and chemotherapy and as such are being investigated for their role in tumor recurrence..$^{8,9}$

\section{Glioma cellular heterogeneity}

Light microscopic studies have shown that tumors that develop within normal tissues comprise a heterogeneous collection of cell types, frequently including immune cells, stromal cells (mesenchymal and endothelial cells), and a variety of normal or malignant cells specific to the tissue. ${ }^{10,11}$ Further, cells within the tumor often seem to correspond to different stages of development. The prevailing explanation for tumor cell heterogeneity is a consequence of influences of the microenvironment and genomic instability that generate genetic and epigenetic changes. ${ }^{12}$ As a result, these factors prevent faithful and accurate replication and transmission of stable genotypes and phenotypes. Alternatively, an emerging concept poses that malignant cell populations may reflect the continuing operation of aberrant and malignant differentiation processes creating heterogeneous tumor phenotypes derived from a small subset of CSCs.

\section{BTSC cell of origin}

In the nascent field of CSCs, many fundamental questions remain unanswered. One critical question is whether these cells arise from NSCs or from more differentiated cells that acquire the capacity of self-renewal. Evidence for the latter exists in the hematopoietic field in blast crisis chronic myelogenous leukemia, in which a committed granulocyte-macrophage progenitor may acquire self-renewal capacity and thus "reacquire" stem-like properties due to the effects of later mutations. ${ }^{13}$ The term CSC is not meant to suggest a stem cell origin, but rather a cancerous cell that has stem cell-like properties, namely extensive self renewal and capacity for differentiation. However, which cells give rise to CSCs remains unknown.

\section{Isolation and characterization of BTSCs}

Expanding on seminal work performed identifying CSCs in the hematopoietic system, ${ }^{14} \mathrm{CSCs}$ in breast cancer were assayed and identified to provide the first evidence of CSCs in solid organ tumors. ${ }^{15}$ Subsequently, it was demonstrated that a subpopulation of cells from glioma could recapitulate the primary glioma with xenograft transplantation of as few as 100 cells. ${ }^{5}$ Currently, CSCs can be best defined experimentally by their ability to perpetuate tumors in serial transplantation xenotransplantation assays in vivo. ${ }^{13}$

An ideal in vitro BTSC assay would employ a highly specific and quantitative guideline, only measuring the cells of interest. In addition, this assay should be sufficiently sensitive so as to be able to measure candidate BTSCs when present at very low frequency. A few examples of in vitro assays currently in use to enrich for CSCs include: sphere, serial colony-forming unit (CFU) and label-retention (BrdU) assays. ${ }^{16}$ Another technique, the side population (SP) phenotype (defined by its ability to efflux the nucleic acid-staining dye Hoechst) has been used to enrich for BTSCs. ${ }^{16-18}$

Flow sorting (fluorescence-activated cell sorting) techniques to prospectively isolate BTSCs on the basis of cell surface markers that characterize and define bona fide CSCs would be ideal for cellular, molecular, and clinical investigation. Presently, the BTSC subpopulation can be enriched for by sorting for the cell surface marker CD133+ (Prominin-1). ${ }^{19,20}$ However, this marker is not an unequivocal marker that can be used for prospective isolation of BTSCs in the brain. In fact, recent studies suggest that CD133- cells may also give rise to brain tumors..$^{21,22}$

Recently, the contributions of the microenvironment have shown that BTSCs exist in a perivascular niche by providing evidence that the microvasculature forms scaffolding that may be necessary for maintaining or promoting the CSCs. Also, it has been shown that in the Patched mutant mouse (model of medulloblastoma), are not propagated by CD133+ cells but by cells expressing the progenitor markers Math1 and CD15/SSEA-1.23 These cells have a distinct expression profile that suggests increased proliferative capacity and decreased tendency to undergo apoptosis and differentiation. CD15 was also found in a subset of human medulloblastomas, and tumors expressing genes similar to those found in murine $\mathrm{CD} 15^{+}$cells have a poorer prognosis. Therefore, CD15 may represent an important marker for CSCs in medulloblastoma. ${ }^{24,25}$ However, it clearly does not suffice to define CSCs solely on surface markers. To further understand and characterize these aberrant stem cells, it would be prudent to link marker expression and self-renewal capacity with underlying molecular changes.

\section{BTSCs as the source of glioma recurrence}

Unlike other solid organ tumors, gliomas do not metastasize and patients succumb to this disease from invariable tumor 
recurrence. This has been thought to result from insufficient reduction of tumor cell number, with most malignant cells considered competent for tumor formation. ${ }^{26}$

Glioma recurrence is now being understood to arise from a subset of tumor cells that exhibit stem cell-like features, CSCs. Consequently, while current cancer treatments are cytotoxic to the bulk of tumor cells, they fail to eliminate CSCs, thereby making them the leading candidates for the origin of glioma recurrence. To elucidate the biology of recurrent gliomas, we investigated the fraction of CSCs in primary vs recurrent (post treatment) gliomas. Human and mouse CSCs can be isolated by sorting for the cell surface marker CD133+. Although CD133 is not an unequivocal marker useful for prospective isolation, it is a highly reliable marker of malignant glioma CSCs with robust capacity to initiate tumors upon xenotransplantation and can be used to enrich for CSCs as a starting point for investigation. ${ }^{21}$ Our preliminary evidence using human CSCs in the xenotransplantation assay shows that the CSC fraction $\left(\mathrm{CD}_{133^{+}}\right.$cells) increases in recurrent gliomas (unpublished). Moreover, recent work by Bao et al and Piccirillo et al provide new in vitro and in vivo evidence supporting the involvement of CSCs in tumors of the brain (Figure 1). ${ }^{20,27}$ Working with mouse models of glioblastoma,

\section{A Treatment with ionizing radiation}

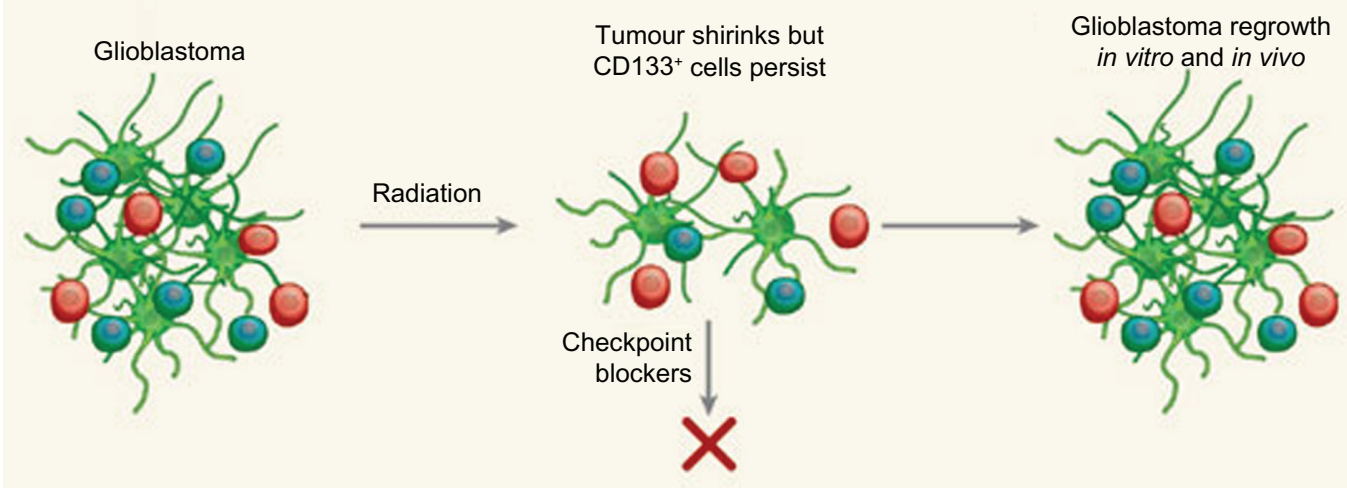

B Treatment with BMPs

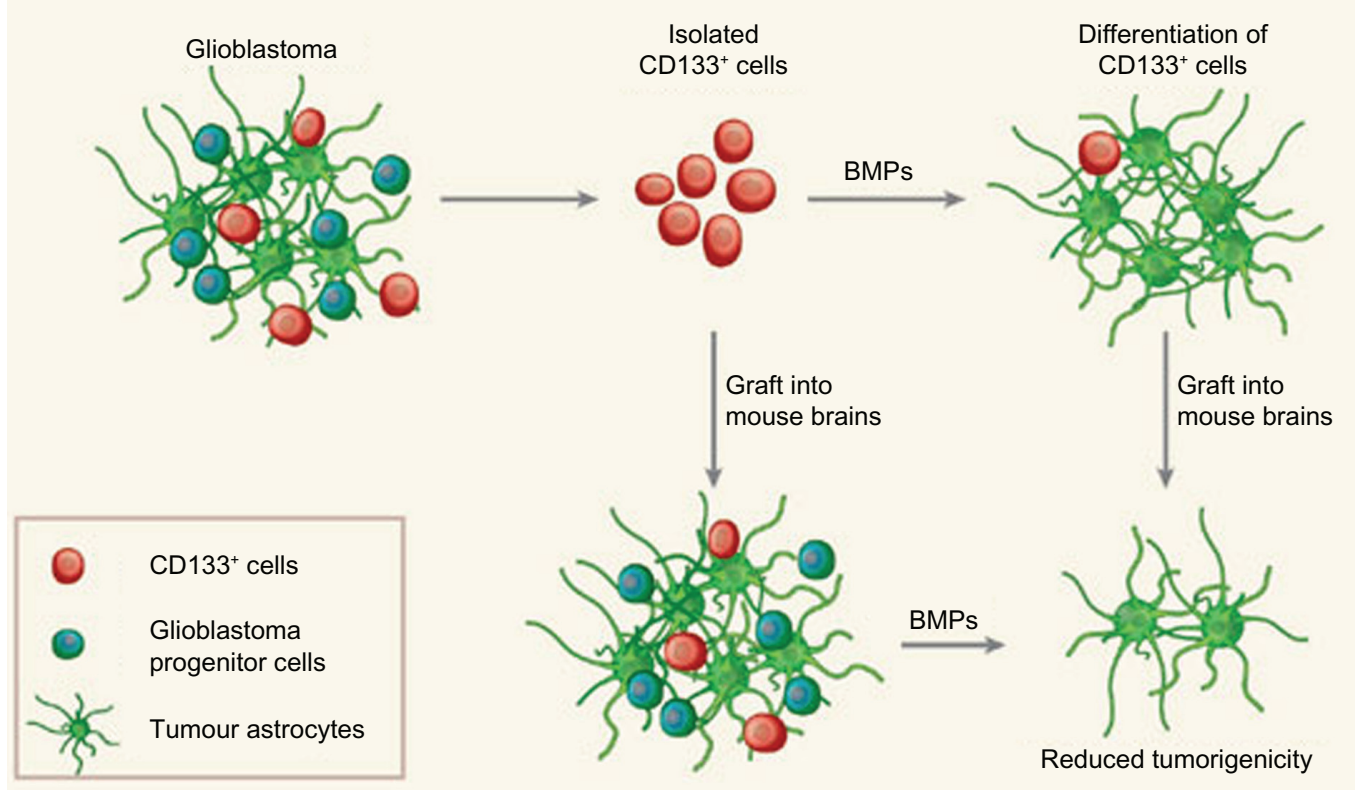

Figure I Glioblastomas are heterogeneous tumors that contain a few tumor-initiating $C D I 33^{+}$stem cells among other, more differentiated, CDI33- cells, including glioblastoma progenitor cells. A) Following radiation, the bulk glioblastoma responds and the tumor shrinks. But CDI33+ cells activate checkpoint controls for DNA repair more strongly than $\mathrm{CDI} 33^{-}$cells, resist radiation and prompt the tumor to regrow. These cells could be targeted with DNA-checkpoint blockers to render them radiosensitive. B) BMPs normally cause NSCs to differentiate into astrocytes. When used to treat isolated glioblastoma CDI $33^{+}$cells, they weaken the cells' tumorigenicity both in vitro and when engrafted into mice, in vivo. The knowledge that a tumor retains a developmental hierarchy suggests that targeting different cell populations is a promising therapeutic strategy. Copyright (c) 2006, Nature Publishing Group. Reprinted with permission from Dirks PB. Cancer: stem cells and brain tumours. Nature. 2006;444 (7|20):687-688.

Abbreviation: BMPs, bone morphogenic proteins. 
the two groups described mechanisms by which BTSC cells escape antitumor treatment and approaches for forcing CSCs to mature to differentiated cells, with consequent loss of self renewal.

\section{Epigenetic instability in gliomas that create CSC competition and clonal diversity}

Along with genetic and molecular mutations, epigenetic alterations also affect the expression of cancer genes and in gliomas multiple microRNAs are aberrantly expressed or repressed in both primary tumors and cell lines. ${ }^{28}$ Overall, microRNAs appear to play multiple roles in gliomas with respect to self renewal and clonal evolution of CSCs. ${ }^{12}$ Moreover, in gliomas, hundreds of genes are subject to DNA hypermethylation at their $\mathrm{CpG}$ island promoters. ${ }^{29}$ A subset of gliomas is also characterized by locus-specific and genome-wide decrease in DNA methylation, or DNA hypomethylation. ${ }^{28}$ Other epigenetic alterations, such as changes in the position of histone variants and changes in histone modifications are also likely to be important in the molecular pathology of gliomas. These epigenetic contributions must also be considered in the successful elucidation of BTSC biology and the possibility that in tumor recurrence BTSCs most likely compete with other BTSCs for dominance in creating tumor recurrence. This possibility is one that would require molecular lineage techniques to follow the clonal evolution and diversity of BTSCs in primary vs recurrent gliomas.

\section{Targeting elusive CSCs}

Insights from other disciplines have led to unanticipated links between CSC biology, neurosciences, and epidemiology. Mutations in neurotransmission pathways including sodium, potassium, and calcium channels have recently been shown to be a common occurrence in gliomas. ${ }^{30}$ These include mutations in all five of the major neurotransmission classes, serotonin, dopamine, acetylcholine, glutamate and GABA. ${ }^{26}$ Interestingly, this suggests that neuromodulatory mutations could confer selective advantage rather than just representing genetic alteration. Furthermore, unlike other mutations (ie, PTEN, P53) discovered in other cancers (ie, pancreases, breast, colon) mutations in neurotransmission pathways appear to be a feature unique to gliomas. ${ }^{31}$ These mutually exclusive cancer driving mutations may thus be a consequence of the unique mechanism these pathways play in regulating CSCs and neural precursor pool size and tendency to differentiate.
Such perturbations in basal neurotransmission inputs from the surrounding NSC niche may be enough to change the dynamics of the CSCs and NSCs pool. This activation of normal lineage-specific signaling pathways in neural precursor derived cancer cells may provide a means of depleting the undifferentiated pool of cells that initiate and maintain brain tumor growth (Figure 2). For example, it has recently been demonstrated that pro-differentiating agents such as bone morphogenic proteins (BMPs) can promote the differentiation of gliomas CSCs and prolong the life of mice xenotransplanted with human tumors. Moreover, in vivo neuromodulation of the metabotropic glutamate receptor 4 (mGlu4), a candidate target for the treatment of Parkinson's disease, has also been shown to attenuate medulloblastoma formation in mice. Similarly, mGlu2/3 antagonism also suppresses both the in vitro and in vivo growth of human glioma cells. $^{30}$

The generality of this phenomenon is suggested by the finding that a broad spectrum of drugs that act on diverse neurotransmission pathways inhibit both normal and cancerous neural precursor cell proliferation in vitro. Based on a high through put screening of 1257 potential agents, the most potent NSCs and potentially anti-CSCs agents identified in these in vitro screens included the clinically prescribed dopamine agonist apomorphine and the glutamate antagonist ifenprodil. ${ }^{32}$ Thus, chronic administration of neuromodulatory agents may deplete CSCs pools in the neuropsychiatric patient and thereby protect against cancer initiation, perpetuation, or recurrence. This group of well tolerated neuropharmacological agents used in standard clinical practice may offer the prospect of rapid new clinical application following clinical trials in patients with brain cancer.

\section{Conclusion}

The immediate goal at hand should be a consensus identification of the elusive CSCs. This objective is achieved through the integration and analysis of multiple methods including functional assays, marker analysis, and analysis of genetic and epigenetic signatures. Subsequently, this will allow for the isolation and characterization of these cells and a comparison to their normal stem cells counterparts. In understanding CSCs development and biology, we have the possibility of generating novel targets that could overcome issues of drug resistance, improve therapeutic efficacy, and decrease tumor recurrence.

\section{Disclosures}

The authors report no conflicts of interest in this work. 
A
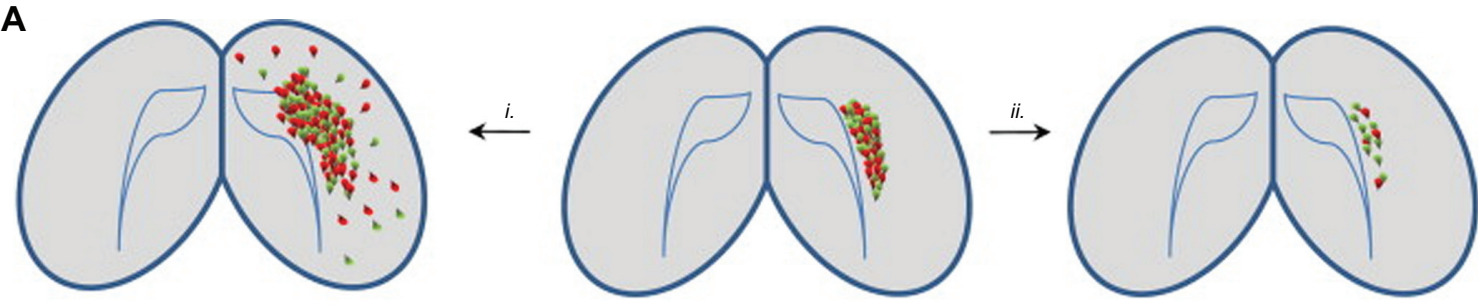

Relative risk of brain tumors

size of precursor compartment

B

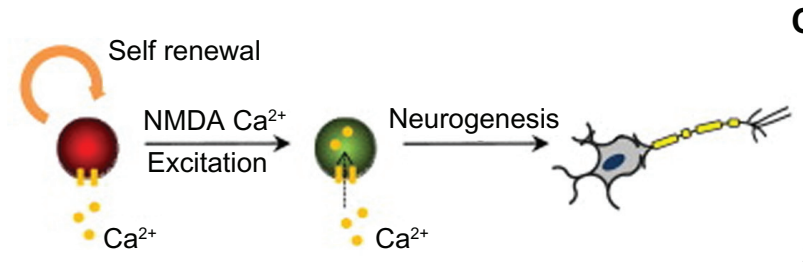

C GABAergic Dopaminergic

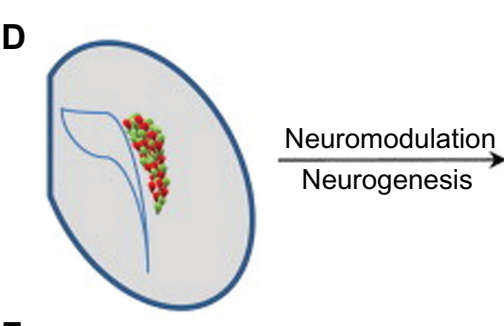

$\mathbf{F}$

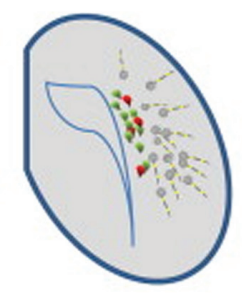

E GABAergic Dopaminergic

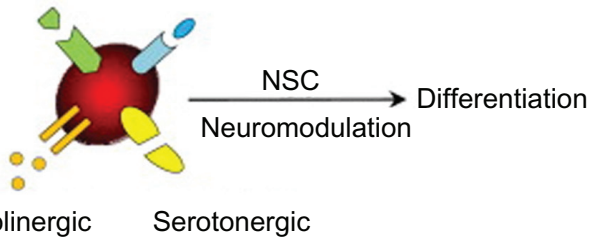

Cholinergic Serotonergic

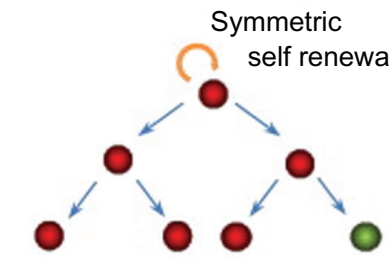

NSC expansion

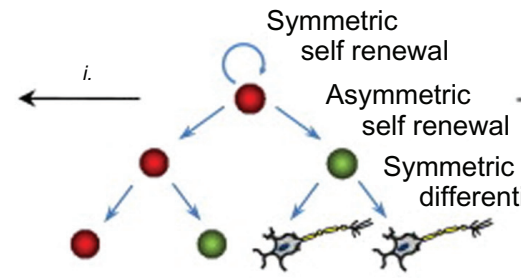

NSC maintenance

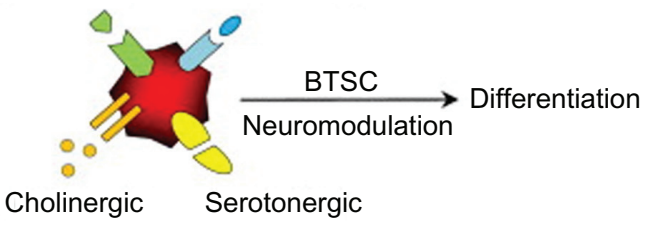

Cholinergic Serotonergic

Figure 2 Neural precursor compartments and brain cancer. A) The mature adult brain contains remnant populations of undifferentiated NSCs in the dentate gyrus and subventricular zone (center panel). (i) Agents leading to the expansion of this compartment (eg, EGF, oncogenic viruses) cause cancer-like lesions in the brain. (ii) Factors reducing the size of this compartment may thus reduce the incidence of brain cancer. B) Like mature glutaminergic neurons, NSCs have been shown to express and respond to NMDA excitatory stimuli, resulting in the expression of differentiation programs and the generation of mature functional neurons. C) Neurotransmitter-induced neurogenesis also occurs following dopaminergic, GABAergic, serotonergic and cholinergic stimuli. D) The initiation of neuronal-specific differentiation of neural precursors occurs at clinically relevant dosages of neuromodulators (eg, haloperidol) in mouse models. E) Like their normal NSC counterparts, the promiscuous expression and responsiveness to neurotransmission are also conserved in cancer-derived neural precursor populations. F) NSC compartments in the adult brain are maintained by symmetric and asymmetric cell divisions. (i) a bias towards symmetric divisions (eg, increased EGF signaling) may expand these pools and lead to cancer. (ii) Signals favoring precursor differentiation (eg, bone morphogenic protein, neuromodulators), may lead to the depletion of the self-renewing population and suppression of brain cancer initiation. Copyright @ 2009, Elsevier. Reprinted with permission from Diamandis P, Sacher AG, Tyers M, Dirks PB. New drugs for brain tumors? Insights from chemical probing of neural stem cells. Med Hypotheses. 2009;72(6):683-687.

Abbreviations: NSCs, neural stem cells; EGF, epidermal growth factor; BTSC, brain tumor stem cells.

\section{References}

1. Stiles CD, Rowitch DH. Glioma stem cells: a midterm exam. Neuron. 2008;58(6):832-846.

2. Szabo E. Selecting targets for cancer prevention: where do we go from here? Nat Rev Cancer. 2006;6(11):867-874.

3. Johannessen TC, Bjerkvig R, Tysnes BB. DNA repair and cancer stem-like cells - potential partners in glioma drug resistance? Cancer Treat Rev. 2008;34(6):558-567.
4. Buzzeo MP, Scott EW, Cogle CR. The hunt for cancer-initiating cells: a history stemming from leukemia. Leukemia. 2007;21(8): $1619-1627$.

5. Singh SK, Hawkins C, Clarke ID, et al. Identification of human brain tumour initiating cells. Nature. 2004;432(7015): 396-401.

6. Galli R, Binda E, Orfanelli $\mathrm{U}$, et al. Isolation and characterization of tumorigenic, stem-like neural precursors from human glioblastoma. Cancer Res. 2004;64(19):7011-7021. 
7. Fael Al-Mayhani TM, Ball SL, Zhao JW, et al. An efficient method for derivation and propagation of glioblastoma cell lines that conserves the molecular profile of their original tumours. J Neurosci Methods. 2009;176(2):192-199.

8. Dirks PB. Brain tumor stem cells: bringing order to the chaos of brain cancer. J Clin Oncol. 2008;26(17):2916-2924.

9. Chalmers AJ. Radioresistant glioma stem cells - therapeutic obstacle or promising target? DNA Repair. 2007;6(9):1391-1394.

10. Gilbertson RJ, Rich JN. Making a tumour's bed: glioblastoma stem cells and the vascular niche. Nat Rev Cancer. 2007;7(10):733-736.

11. Yang I, Aghi MK. New advances that enable identification of glioblastoma recurrence. Nat Rev Clin Oncol. 2009;6(11):648-657.

12. Lagasse E. Cancer stem cells with genetic instability: the best vehicle with the best engine for cancer. Gene Ther. 2008;15(2):136-142.

13. Clarke MF, Dick JE, Dirks PB, et al. Cancer stem cells - perspectives on current status and future directions: AACR Workshop on cancer stem cells. Cancer Res. 2006;66(19):9339-9344.

14. Bonnet D, Dick JE. Human acute myeloid leukemia is organized as a hierarchy that originates from a primitive hematopoietic cell. Nat Med. 1997;3(7):730-737.

15. Al-Hajj M, Wicha MS, Benito-Hernandez A, Morrison SJ, Clarke MF. Prospective identification of tumorigenic breast cancer cells. Proc Nat Acad Sci U S A. 2003;100(7):3983-3988.

16. Teicher BA. In vivo/ex vivo and in situ assays used in cancer research: a brief review. Toxicol Pathol. 2009;37(1):114-122.

17. Zhang P, Lathia JD, Flavahan WA, Rich JN, Mattson MP. Squelching glioblastoma stem cells by targeting REST for proteasomal degradation. Trends Neurosci. 2009;32(11):559-565.

18. Fukaya R, Ohta S, Yamaguchi M, et al. Isolation of cancer stem-like cells from a side population of a human glioblastoma cell line, SK-MG-1. Cancer Lett. 2009.

19. Nishide K, Nakatani Y, Kiyonari H, Kondo T. Glioblastoma formation from cell population depleted of Prominin1-expressing cells. PloS One. 2009;4(8):e6869.

20. Bao S, Wu Q, McLendon RE, et al. Glioma stem cells promote radioresistance by preferential activation of the DNA damage response. Nature. 2006;444(7120):756-760.
21. Clement V, Dutoit V, Marino D, Dietrich PY, Radovanovic I. Limits of CD133 as a marker of glioma self-renewing cells. Int J Cancer. 2009;125(1):244-248.

22. Beier D, Hau P, Proescholdt M, et al. CD133(+) and CD133(-) glioblastomaderived cancer stem cells show differential growth characteristics and molecular profiles. Cancer Res. 2007;67(9):4010-4015.

23. Ward RJ, Lee L, Graham K, et al. Multipotent CD15+ cancer stem cells in patched-1-deficient mouse medulloblastoma. Cancer Res. 2009;69(11):4682-4690.

24. Panchision DM, Chen HL, Pistollato F, Papini D, Ni HT, Hawley TS. Optimized flow cytometric analysis of central nervous system tissue reveals novel functional relationships among cells expressing CD133, CD15, and CD24. Stem Cells. 2007;25(6):1560-1570.

25. Read TA, Fogarty MP, Markant SL, et al. Identification of CD15 as a marker for tumor-propagating cells in a mouse model of medulloblastoma. Cancer Cell. 2009;15(2):135-147.

26. Zhou BB, Zhang H, Damelin M, Geles KG, Grindley JC, Dirks PB. Tumour-initiating cells: challenges and opportunities for anticancer drug discovery. Nat Rev Drug Discov. 2009;8(10):806-823.

27. Piccirillo SG, Reynolds BA, Zanetti N, et al. Bone morphogenetic proteins inhibit the tumorigenic potential of human brain tumourinitiating cells. Nature. 2006;444(7120):761-765.

28. Nagarajan RP, Costello JF. Epigenetic mechanisms in glioblastoma multiforme. Semin Cancer Biol. 2009;19(3):188-197.

29. Rajasekhar VK, Dalerba P, Passegue E, Lagasse E, Najbauer J. The 5th International Society for Stem Cell Research (ISSCR) Annual Meeting, June 2007. Stem Cells. 2008;26(1):292-298.

30. Diamandis P, Sacher AG, Tyers M, Dirks PB. New drugs for brain tumors? Insights from chemical probing of neural stem cells. Med Hypotheses. 2009;72(6):683-687.

31. Parsons DW, Jones S, Zhang X, etal.Anintegrated genomic analysis of human glioblastoma multiforme. Science. 2008;321(5897):1807-1812.

32. Diamandis P, Wildenhain J, Clarke ID, et al. Chemical genetics reveals a complex functional ground state of neural stem cells. Nat Chem Biol. 2007;3(5):268-273.

33. Dirks PB. Cancer: stem cells and brain tumours. Nature. 2006;444 (7120):687-688.
Biologics: Targets \& Therapy

\section{Publish your work in this journal}

Biologics: Targets \& Therapy is an international, peer-reviewed journal focusing on the patho-physiological rationale for and clinical application of Biologic agents in the management of autoimmune diseases, cancers or other pathologies where a molecular target can be identified. This journal is indexed on PubMed Central, CAS, EMBase, Scopus

\section{Dovepress}

and the Elsevier Bibliographic databases. The manuscript management system is completely online and includes a very quick and fair peerreview system, which is all easy to use. Visit http://www.dovepress. com/testimonials.php to read real quotes from published authors. 